\title{
Current Advances in Anti-Influenza Therapy
}

R. Saladino*,1, M. Barontini1, M. Crucianelli2, L. Nencioni3, R. Sgarbanti3 and A.T. Palamara4,5 IDipartimento di Agrobiologia ed Agrochimica, University of Tuscia, Via S. Camillo de Lellis snc, 01100 Viterbo, Italy; ${ }_{2}$ Dipartimento di Chimica, Ingegneria Chimica e Materiali, University of L'Aquila, via Vetoio, 67100 Coppito (AQ), Italy; 3 Dipartimento di Scienze di Sanità Pubblica, "Sapienza" University of Rome, 00185 Rome, Italy; 4 Istituto Pasteur, Fondazione Cenci Bolognetti, Dipartimento di Scienze di Sanità Pubblica, University of Rome, 00185 Rome, Italy; sIRCSS San Raffaele La Pisana, Rome, Italy 